Review

\title{
Nano-Scaled Particles and Fibres Occupational Exposure Assessment: An Integrated Approach from Air Sampling to Skin and Surface Contamination
}

\author{
Andrea Prodi ${ }^{`}$, Francesca Larese Filon \\ University of Trieste, Clinical Unit of Occupational Medicine, via della Pietà 19, 34129 Trieste. \\ Corresponding author. E-mail: andrea.prodi@phd.units.it
}

Received: May 16, 2016; Accepted: Jun. 15, 2016; Published: Jun. 27, 2016

Citation: Andrea Prodi, Francesca Larese Filon, Nano-Scaled Particles and Fibres Occupational Exposure Assessment: An Integrated Approach from Air Sampling to Skin and Surface Contamination. Nano Biomed. Eng., 2016, 8(2): 91-I04.

DOI: 10.5101/nbe.v8i2.p91-104.

\begin{abstract}
Workers may be exposed to nanoparticles (NPs) by inhalation, cutaneous contact and gastroenteric pathways, but today there is no standardized method for either assessing or monitoring the occupational exposure. Moreover, there is no all-in-one assessment strategy, so it is preferable to consider different perspectives. Every assessment should be preceded by a preliminary analysis of the workplace in order to gather useful data on the potential exposure sources, which will help in outlining the assessment strategy. The purpose of air monitoring should be to characterize NP emissions and assess, as a minimum, the mass, particle number concentration, granulometric distribution, specific surface and chemical composition. There are several techniques which may assess these characteristics: the most used in real occupational contexts are mobility particle sizers, particle counters (optical, condensation, etc.), surface area monitors, while personal samplers are promising tools which still have been used almost only in research or controlled contexts. Skin and surfaces may be sampled according to the nature of substances and the circumstances of exposure. Sampling methods can be divided in three main categories: interception (e.g. carbon tabs), removal (e.g. adhesive tape stripping, wiping), in situ methods (e.g. UV fluorescence). Chemical analysis methods (e.g. spectroscopy) and electron microscopy techniques may enhance available data. Considering the available information, we suggest a stepwise approach for risk assessment composed of three steps, starting from a quick and relatively cheap screening method to assess exposure, followed by 2 gradually more accurate but costly approaches to perform whether a significant exposure is detected.
\end{abstract}

Keywords: Nanoparticles; Nanomaterials; Risk assessment; Air sampling; Contamination; Skin; Surfaces; Workplace; Occupational health

\section{Introduction}

Nanoparticles (NPs) production and use is increasing worldwide due to novel interesting applications in electronics, medicine, cosmetics, textiles, semiconductors, varnishes, surface treatments, etc. Many benefits are expected from their use but potential toxic effects related to NPs exposures have been highlighted [1-4]. Due to their small size NPs are able to enter into our body. Through inhalation route they can damage the lung, some of them can translocate to the bloodstream reaching other organs 
and tissues at cellular and molecular level. NPs may access to the brain through nose or passing the bloodbrain barrier. NPs can also penetrate and permeate the skin, mainly if damaged [5].

For that reason it is extremely important to avoid NPs exposure using "safe by design" techniques, close systems, wet formulations, effective local exhaustion and protective equipment. Moreover it is important to evaluate NPs exposure to verify working condition and protect workers to avoid short and long term effects.

The exposure of workers to nanoparticles (NPs) may follow several routes: inhalation, cutaneous contact and gastroenteric pathway (inadvertent ingestion from contact between contaminated hands or objects and the mouth).

The respiratory path is by far the most studied. The exposure can occur at each stage of the production cycle, and it is important to limit the occupational exposure reducing the presence of free airborne NPs.

This may be done by encapsulating emission sources, choosing low dust emission formulations of raw materials, intermediate and final products, adopting fume hood, draw benches, proper ventilation and setting adequate strategies for the management of exhausts. It is also fundamental to give workers proper personal protective devices. Emitted NPs can be deposited on different surfaces, equipment, clothing and on the skin, creating an important potential secondary source of airborne NPs (e.g. by resuspension) [6].

The contamination from NPs and their interaction with the skin layers are a matter of investigation of increasing importance in occupational health research. Several studies have demonstrated that some nanocompounds can penetrate the outer layers of the stratum corneum while others can permeate and cross the dermal layer (by the intercellular and/or intracellular route or passing through the skin annexes), reaching the systemic circulation.

The lipid matrix of the stratum corneum and of the skin annexes can also act as a significant reservoir for penetrated chemicals and as an intermediate step in reaching blood stream.

The individual characteristics (gender, skin thickness, hair follicle density, blood flow, age, mechanical flexion of skin, systemic diseases, etc.) as well as damages to the stratum corneum (e.g. by irritant compounds, skin diseases, atopic eczema, etc.) can increase the probability of absorption.

Intrinsic parameters of NPs (composition, size, shape, surface chemistry, etc.) may significantly be influenced by the properties of the physiological media and of the environment (background factors, vehicle, density, temperature, $\mathrm{pH}$, etc.), altering the behaviour of NPs itself, in particular causing aggregation, agglomeration (reaching non-nano sized dimensions), precipitation.

Three main aspects are important in the evaluation of the occupational safety profiles [5]:

(a) Size: the smaller the particle, the higher are the probability to cross the skin, especially in impaired skin barrier conditions. It is important to consider that most of the NPs tend to aggregate or disaggregate in real conditions, changing their global size; this behaviour is influenced by the physiological media or by the environmental conditions.

(b) Composition and capacity to release ions and/or toxic compounds: NPs containing metals can release a greater amount of ions compared to bulk materials, given their higher surface mass ratio. This release may increase the risk of local and systemic toxic effects such as skin and/or airways sensitization, especially in presence of high sensitizing metals such as nickel, palladium, chromium and cobalt.

(c) Protective measures of workers: risk can be influenced by factors such as skin flexion and/or skin barrier impairment, use of protective equipment, the reduction of workplace contamination, proper information on risks, etc.

At present, there are no existing standardized methods for either assessing or monitoring the occupational exposure to nanoparticles [7]. The methods presented in this review are commonly chosen assessment strategies and have either been used in several occupational contexts for the study of nanoparticles or may be adapted to.

The contamination of surfaces and skin may influence the airborne presence of nanoparticles and vice-versa. Skin exposure is also worth evaluation in order to better understand whether workers can come in contact with nanoparticles via this exposure route. Given all this considerations, an integrated approach which takes into account air sampling and skin and surface contamination should be recommended. Graph 1 shows a summary of existing techniques for risk assessment. 


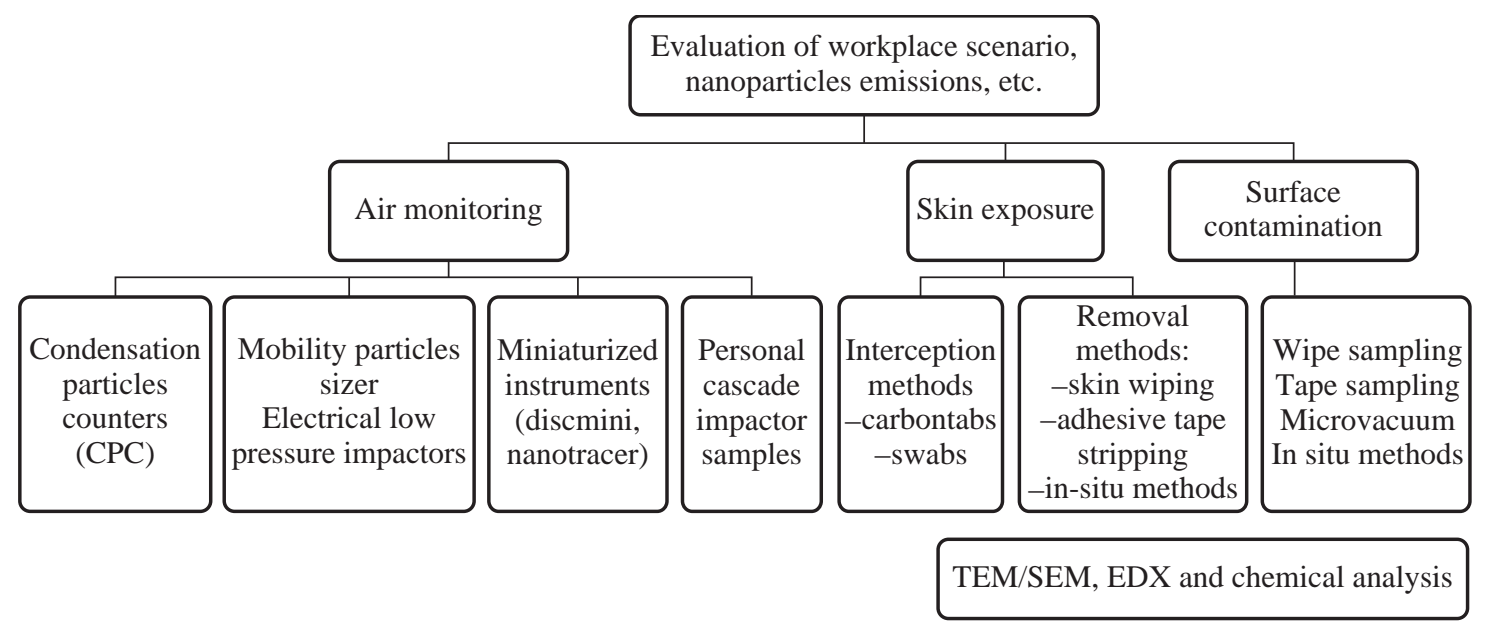

Graph 1 Summary of the techniques and approaches for risk assessment.

\section{General Recommendation Prior to Each Assessment}

Before proceeding with quantitative assessments, a qualitative assessment should be performed in each workplace scenario in order to gather data regarding the production facilities and methods:

- Raw materials, intermediate and final products, polluting substances originated during production;

- The source and the size of expected nanoparticle emissions;

- Number and task of workers involved in the production and the exposure times for each task;

- Information about workers' garments and personal protective devices;

- Planimetry of the production facility, ventilation (and the direction of air flows) and the presence of aspiration devices;

- Location, type of machinery and their exact role in the productive process;

- The geometry and roughness of surfaces in the workplace;

- The cleaning procedures of the workplace, workers and the management of wastes.

Proper information about the assessment practices should be given to the workers and to the company leadership.

\section{Air Monitoring for Nanoparticles}

The air monitoring strategy for nanoparticles may adapt techniques already used for non-nano scaled compounds (such as personal and environmental samplers) and/or utilize newly developed instruments. At present there is no all-in-one solution but instead it is preferable to take into consideration different points of view. There is currently no consensus concerning ultrafine particles and NPs exposure evaluation measures [8].

The purpose of air monitoring should be to characterize NP emissions and assess, as a minimum, the particle number concentration, granulometric distribution, specific surface and chemical composition [9]. There are several techniques which may assess the mass and/or the number of particles and/or the specific surface and the granulometric distributions of the NPs.

Some examples of techniques and instruments useful for NP aerosol assessment [8]:

- Condensation particle counters (CPC) allows the determination of the particle number concentration and real time concentration of fine and ultrafine particles; other particle counters such as OPC (Optical Particle Counter) have also been used.

- Electrical Low Pressure Impactors (ELPI), Mobility Particle Sizer (such as Scanning Mobility Particle Sizer, SMPS): those techniques can determine a set of parameters such as numerical, mass and specific granulometric distributions.

- Newly developed miniaturized instruments: which has the advantage to be placed in the respiratory zone, such as passive aerosol dosimeters [10], Aerasense Phillips NanoTracer ${ }^{\circledR}[11,12]$, DiSCMini Diffusion Size Classifier, thermal precipitators [13, 14].

The particles measurements may be performed with 
various instrumentation, placing it at the emission source (electrical mobility spectrometers - e.g.: SMPS -, optical particle sizers, condensation particle counters, etc.) or in the worker's breathing zone (optical particle counter, light-scattering laser photometers, miniature diffusion size classifiers - e.g. DiSCMini -, etc.).

As for other chemicals air monitoring, samples may be collected for further type of studies (chemical analysis, EDX spectroscopy, mass determination, etc.). Sampling cassettes with the use of filters (e.g. polycarbonate filters) and pumps can be used to perform environmental samples while personal cascade impactor samplers, with a proper collection substrate (e.g. quartz fibres filters, Teflon-PTFE filters) can be applied individually on workers [15].

The filters may subsequently undergo chemical analysis, e.g. by chemical digestion and subsequent ICP analysis (ICP-AES, ICP-MS, etc.).

Mass concentration can be gravimetrically determined by pre- and post-weighting the filters on a microbalance. Particle morphology, chemical composition and size may be analysed by means of scanning electron microscope or transmission electron microscope, coupled with an energy dispersive X-ray (EDX) spectroscopy.

\section{Assessment of Skin Exposure to Nanoparticles and Skin Contamination}

The factors which are able to influence skin exposure are linked [16, 17]:

- to skin's physiological characteristics (body site, sex, age) and skin conditions (impaired stratum corneum, skin hydration, vasodilation, temperature);

- to chemical and physical characteristics of the substance to which workers are exposed (lipophilicity, polarity, chemical structure, volatility, concentration);

- to exposure-related factors (job, exposure time, environmental conditions, skin exposed area, substance concentration, use of garments and personal protective devices, individual hygienic conditions).

The skin may be sampled according to the nature of the substance and the circumstances of the exposure. Those methods can be divided in three main categories: interception (e. g. carbon tabs), removal (e.g. tape lifting) and in situ methods (for fluorescent or UV absorbent substances) $[6,18]$.

\section{Interception methods}

Interception methods are intended to intercept nanoparticles in their route towards the skin, before reaching the outer skin layers.

One of the most promising involves the use of carbon tabs, which are double-sided adhesive tabs used as an interception sampler, fastened to the outside of clothes or directly over the skin. These tabs are useful to sample powders, suitable for viewing in a high vacuum SEM and allowing also analysis on size distribution. They can also play a role in testing the efficacy of personal protective equipment by placing them between the protective equipment and workers' skin.

Carbon tabs should be applied at the beginning of the work shift and removed at the end of a full work shift of 8 hours, positioned:

- Over the garments or protecting devices (gloves, masks): dorsum hands (volar hands may be assessed but usually is not possible), dorsal and volar forearms, face, chest, thighs, feet;

- Under the garments or personal protecting devices, in the same body areas as above.

An estimation of the contamination of workers' skin can be performed using anatomical models [19] and/or mathematical formulas [20].

The manipulation of tabs should be performed carefully, avoiding further contamination or loss of deposited substance. Tabs should be collected and stocked in proper containers and can be further analysed by Scanning Electronic Microscopy (SEM) for gaining quantitative and morphological information on aggregates of particles (Fig. 1) and may be qualitative analysed by complementary techniques such as Energy Dispersive X-Ray analysis (EDX) (Fig. 2).

Given the possibility of skin irritation after long term application of adhesive carbon tabs we would recommend to avoid prolonged direct application on skin but instead to either apply the pads over the skin with an interposition layer (e.g. a garment, an underglove or an armband) or either adopt other interception strategies.

The alternative strategies which might be used can involve the use of other sampling supports, such 


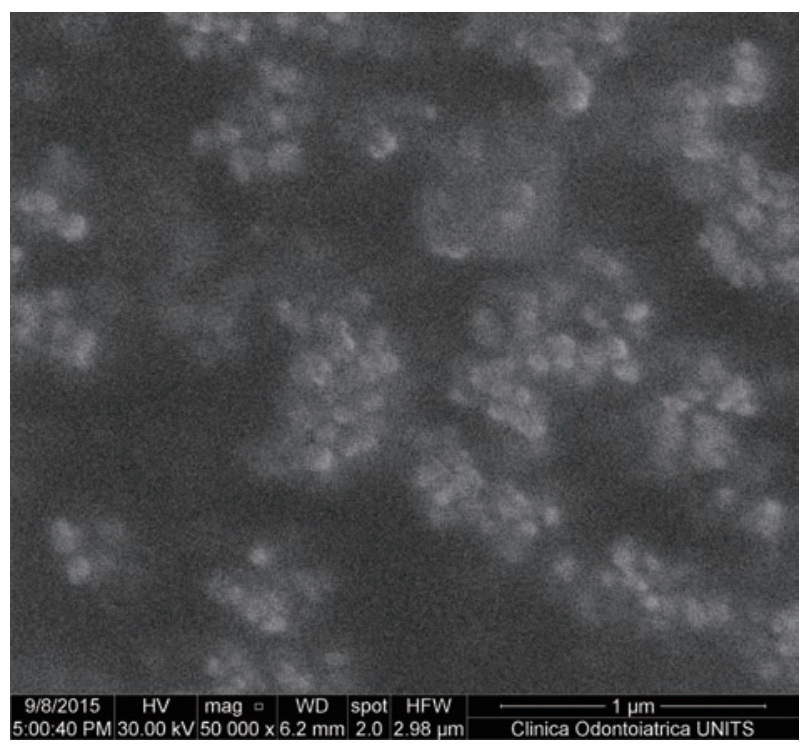

Fig. 1 Nano-sized (< $100 \mathrm{~nm}$ in diameter) aggregates of Aluminium Oxide on a carbon tab from a soldering facilities on aluminium - made goods (sample collected by the author).

as swabs, which can be chemically analysed after collection. It is important in this case to carefully choose the swab, possibly with the consultancy of the laboratory which is going to analyse the swab, since the analytical method may be influenced by the composition of the swab itself and by the chemical nature of the nanoparticles.

\section{Removal methods}

There are several potential approaches in skin assessment by means of removal methods: in particular, the most common are skin wiping and adhesive tape stripping. Another interesting method for dermal exposure assessment is bag rinsing [21] but - at our knowledge - this approach still has not been proposed for nanoparticles.

\section{Skin wiping}

Skin wiping technique consists in the removal of contamination from the skin by swiping it through a swab soaked with water or other skin-safe solvents as needed. The removal must provide, manually, an external force that equals or exceeds the force of adhesion of the assessed material over a defined skin surface area. The contaminant is detached from the skin by a combination of mechanical forces and wet chemical action (dissolution) [21].

We suggest to assess the most exposed skin areas (usually hands, forearms and the face) of selected workers, taking note of the presence of garments and/ or personal protective equipment. The results should be expressed in terms of mass per surface unit, and, whenever possible, a standardized shaped area should be used. We suggest to apply a standardized assessment technique, such as to adapt to the skin the standardized ASTM procedure for surface testing [22]. In this procedure, skin is wiped in three passes, each time using an unexposed surface of the wipe: the first pass consists of s-shaped parallel horizontal movements, the second one of parallel vertical movements (perpendicular to the first pass) while the third pass is made along the perimeter. It is important to take note of the total exposure time of the selected workers.

The tested workers should clean the chosen skin surfaces at the beginning of the work-shift and avoid cleaning them again until final assessment. If this is not possible (e.g. hands for hygienic reasons during lunch), the assessment should be done before skin cleaning (and evaluated taking into consideration the reduced exposure time). To reduce inter-operator variability, it is preferable that only one operator performs the assessment in the whole test, using a new pair of powder less gloves for each sample.

After the sampling procedure, each swab should be placed into a clean container, securely sealed and labelled and stored at environmental temperature before chemical analysis (swabs should be chosen with the consultancy of the laboratory which is going to analyse them, since the analytical method may be influenced by the composition of the swab and by the chemical nature of the NPs). It is also possible to consider the use of special wipes (such as Ghost Wipes), which are easier to dissolve prior to a chemical analysis and have been already used in NPs assessment [18].

\section{Adhesive tape stripping}

Tape stripping allows the removal of the cell layers of the stratum corneum and contaminating particles from the human skin. The protocol of tape stripping may be derived from the international literature, e.g. Lademann J et al. [23]. Strips should be collected at the end of the workday from skin exposed areas of the palmar portion of forearm or forehead, choosing areas with a low amount of hair follicles (the selected areas should be clean at the beginning of the work-shift). The presence of garments or personal protective equipment must be annotated.

Tape stripping is a commonly and successfully used 


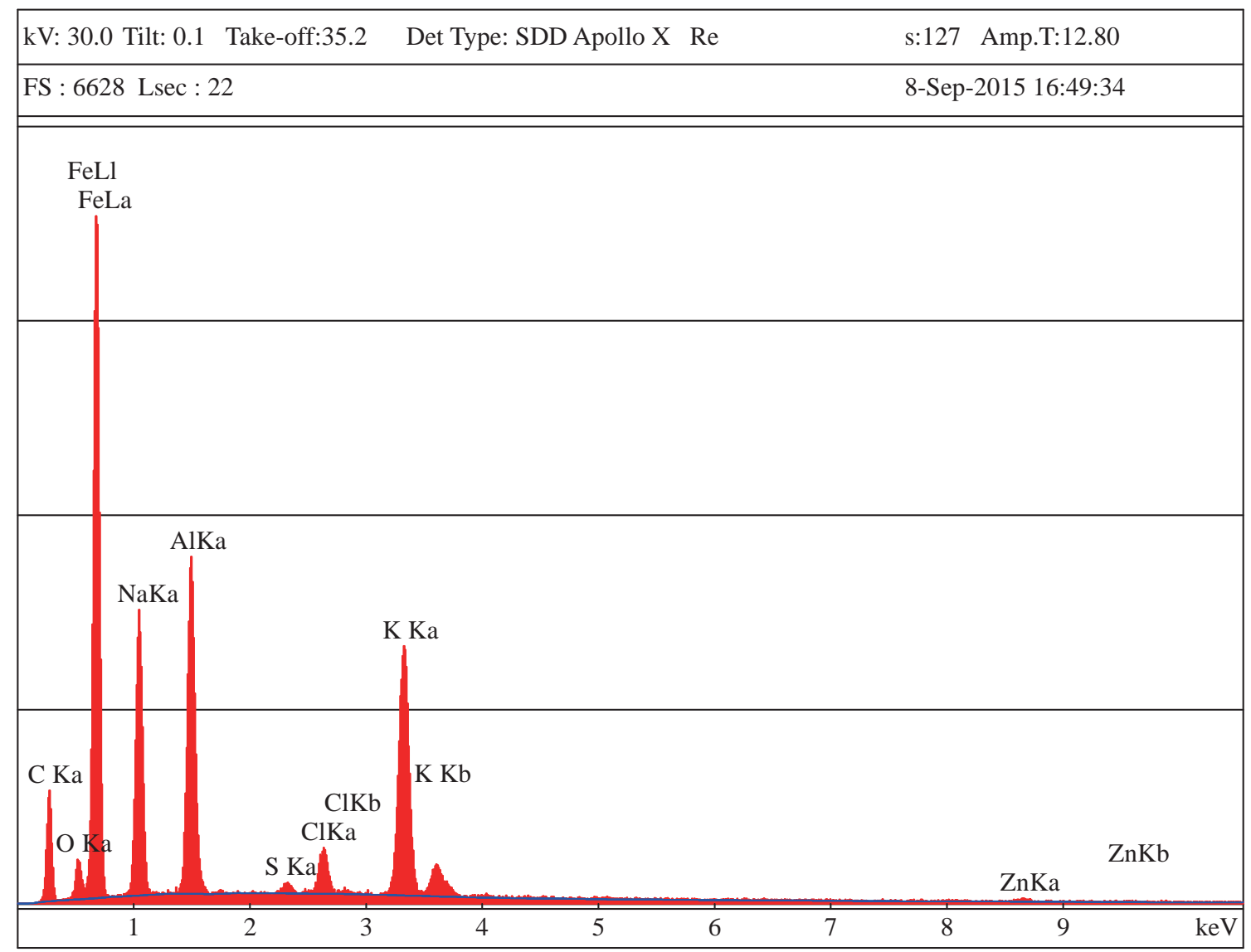

\begin{tabular}{llllll}
\multicolumn{6}{l}{ EDAX ZAF Quantification (Standardless) } \\
\multicolumn{2}{l}{ Element Normalized } \\
SEC Table : Default
\end{tabular}

\begin{tabular}{|lllll|}
\hline Element & Net Inte. & Bkgd Inte. & Inte. Error & P/B \\
\hline C K & 185.93 & 5.00 & 1.58 & 37.21 \\
O K & 72.12 & 9.33 & 2.78 & 7.73 \\
FeL & 766.40 & 12.73 & 0.77 & 60.18 \\
NaK & 592.01 & 21.14 & 0.89 & 28.01 \\
AlK & 780.29 & 29.23 & 0.78 & 26.70 \\
S K & 27.50 & 34.40 & 7.50 & 0.80 \\
ClK & 132.43 & 36.70 & 2.28 & 3.61 \\
K K & 752.12 & 30.42 & 0.80 & 24.72 \\
ZnK & 12.73 & 11.32 & 9.82 & 1.13 \\
\hline
\end{tabular}

Fig. 2 An example of EDX output from a carbon tab applied on a surface in a metalworking facility producing aluminium made goods (sample collected by the author).

technique to study the stratum corneum and to assess the quality and efficacy of cosmetic and dermatologic products and may be adapted to the study of nanoparticles permeation through the stratum corneum.
However, the use of a full tape stripping protocol may be difficult in an occupational context: the protocol requires a long amount of time, a well-trained operator, technical appliances to determine the right 
amount of strips for each tested worker and may cause temporary discomfort to the tested volunteer (redness of skin, itching, skin colour alteration) which might last for some days/weeks after the procedure until the stratum corneum is fully restored.

An approach which can lead to a good approximation of the contamination, limiting most of the issues of a full-scaled protocol, may involve the application of some tape strips (e.g. 1-3) just to assess the external stratum corneum contamination.

Tape strips can be chemically analysed in order to quantify and qualify the contamination and may be studied by SEM / EDX techniques.

\section{In situ methods}

These methods usually take advantage of the fluorescent properties of substances under longwave ultraviolet light (UVA), allowing to a direct visualization on the surfaces (or on the skin).

A previously calibrated software is usually utilized to compare the fluorescent brightness of the substances on the surface and to estimate its total amount.

This is a feature of a limited number of substances, so the variety of particles that may be analysed is reduced, compared to other methods. Other drawbacks of the methodology are related to the fact that since it is a wide-scale visualisation, individual nanoparticles cannot be seen on the surface or on the skin and the particle size cannot be determined.

However, an important advantage is that bulk nanoparticles are observed in real conditions, along with their natural physical behaviour (agglomeration, etc.) [24].

\section{Evaluation of Contamination on Workplace Surfaces}

Workplace contamination of surfaces and equipment can be assessed by several methods (e.g. microvacuum, wipe collection, tape sampling, etc.).

\section{Wipe sampling}

The wipe testing of surfaces is a useful methodology which applies to the analogue technique previously described in the wipe testing of the skin. It is recommended to adopt standardized procedures such as the one from ASTM [22], which requires to choose a $10 \mathrm{~cm}^{2}$ surface and assess it via the previously described methodology.

When testing for the deposition of nanomaterials (e.g. an evaluation of deposition during a production cycle) it is mandatory to assess the cleanliness level of the chosen test areas and to clean them with a cleaning solution appropriate to the assessed materials, before the beginning of the production cycle.

Whenever possible, we suggest to assess smooth or slightly rough surfaces because the roughness may damage the swab and lead to the loss of integrity of the swab.

All other considerations made for the wipe sampling of skin are valid also in the case of surfaces.

\section{Tape sampling}

The tape sampling of surfaces involves the removal of the contaminants that have been deposited on the most external layer of a workplace surface (such as a table, a machinery, a tool, etc.).

It is important to properly choose the characteristics of the adhesive tapes in particular regarding the feasibility of further evaluation (chemical analysis, SEM/EDX, etc.).

As recommended for wipe sampling, when testing the deposition of nanomaterials during a production cycle, a preliminary cleaning of the surface is mandatory at the beginning of the cycle.

It is recommended to collect some blank tape samples to check the potential contamination status after the cleaning procedure and to remove any residuals of adhesive after this procedure.

The surface of the tape should be pressed against the workplace surface and rubbed lightly to ensure the adhesion of particles. Then the tape should be pulled off with a fluent movement and stored. In order to minimize between operator variability on these procedures and on skin removing, all procedures should be performed by the same operator.

Tape strips can be chemically analysed in order to quantify and qualify the contamination and may be studied by SEM / EDX techniques.

Adhesive carbon tabs may be an interesting alternative to tape strips even if, at our knowledge, the use of this type of support still has not been described in international literature. 


\section{Microvacuum}

Surface contamination screenings may be conducted by microvacuum technique. These methods, along with wipe sampling methods, are being used in a growing number of workplaces, despite the uncertainty in the findings at the current state of knowledge [18].

Surface microvacuum samples can be collected on filter media which may then be analysed by means of other explained methodologies such as SEM/TEM and energy dispersive X-ray spectroscopy. With such methodologies, the particles and their aggregates can be directly seen and analysed in terms of composition.

It is suggested to use a standardized sampling area (such as $10 \mathrm{~cm}^{2}$ ) whenever possible.

\section{In situ methods}

These methods are similar to the ones already described in the section regarding skin assessment.

\section{Sampling Techniques Used in Real Workplace Contexts for the Assessment of Nanoparticles}

All the above mentioned techniques have been used in workplace assessments for several types of chemicals but, despite the fact that all of them may be readapted for the use in nanoparticle assessment, there still are few international researches on the topic. Some of the most relevant approaches - at our knowledge - are described in the following sections and a more complete reference list is provided in Table 1 at the end of the section. From the table it can be perceived how most of the studies have focused on air monitoring while very few studies have approached the contamination of workers' skin and surfaces.

\section{Air monitoring of nanoparticles}

Several authors have already used techniques for NPs aerosol assessment in both experimental and workplace contexts. In the following paragraph are summarized examples from some of the most recent and relevant articles available on the topic, even though a complete summary would deserve a review on its own.

CPC (Condensation Particle Counters), OPC (Optical Particle Counters) and MPS (Mobility Particle Sizers) are the techniques which have been mostly used in real workplace contexts for the air monitoring of NPs. They have been used for assessing NPs in offices (for laser printers indoor pollution), in semiconductor fabrication, in the production or use of epoxy and/ or carbon nanotubes and nanocomposites, fullerene factories, traditional pottery manufacturing, recycling facilities, industrial use of $\mathrm{TiO}_{2}$, silver nanofibers and several other occupational contexts.

Lee and co-workers [25] have adopted several air monitoring techniques for NPs, such as CPC and SMPS in an occupational context, two facilities conducting research on graphene. Those techniques were used together with other real - time monitoring techniques and with TEM analysis on samples. Researchers found graphene-like structure and aggregated / agglomerated carbon structures in TEM observation, while the mass concentration of suspended particulate and elemental carbon concentrations were very low and mostly below the detection limits.

CPC and SMPS were also used by Niu et al. [26] for assessing particle number concentrations, size distribution, and to characterize the variability of the background aerosols. Performing a point source study of laser printers, authors found that NPs emitted from older models were distinguishable from the background, while emissions from newer printers are likely to be indistinguishable from the background, requiring further chemical characterization to better identify the emission sources.

Shepard and Brenner [27] used CPC, optical particle counters and SMPS in a semiconductor fabrication in which engineered nanoparticles of alumina, amorphous silica and ceria are used during wafer polishing steps in the production of semiconductor devices (the measured concentrations were below currently proposed benchmarks or reference value for poorly soluble low-toxicity nanoparticles). TEM/EDX analysis were furtherly conduced on air samples which identified structures containing the elements of interest $(\mathrm{Al}, \mathrm{Si})$, primarily as agglomerates or aggregates in the 100$1000 \mathrm{~nm}$ size.

The ELPI technique has been used by Kreider et al. [28] in a real workplace environment, successfully managing to assess carbon black and amorphous silica in a tire manufacturing facility, confirming the presence of such NPs in the workplace. The method has been found useful in providing quantitative estimates of worker's exposure to NPs. Using ELPI, Kim et al. [29] have assessed airborne NPs in a rubber 
manufacturing factory which used recycled tires finding a high concentration of specific NPs, including silicon, sulphur and zinc.

Other techniques which are worth to be mentioned are NSAM (Nanoparticle Surface Area Monitors), APS (aerodynamic particle sizers), aerosol monitors, cascade impactors and diffusion chargers. Some examples of occupational contexts in which those methods have been used are listed in Table 1.

Miniaturized instruments are newer innovation among the NPs sampling techniques and there are few international articles on this topic in real occupational contexts. DiSCMini has been found as a reliable tool, but the studies regarding this instrument have been made for environmental or research contexts and not in occupational settings. Along with DiSCMini, also passive aerosol samplers have not been used in occupational contexts for NPs assessment.

\section{Skin contamination}

The adhesive tape stripping technique on the skin of worker, in order to assess skin contamination from nanoparticles, has been adopted by Hedmer et al. [6] testing a worker in a small scale production facility of multi-walled carbon nanotubes. However, the researchers were not able to find nanoparticles within the collected skin layer.

\section{Surfaces contamination}

Shepard and Brenner18 proposed to adopt the ASTM D6480 protocol in the wipe sampling of surfaces in a semiconductor production factory. They found nanoparticles of silica in the surface sampling and silica and/or alumina aggregates. The authors felt that more rigorous and validated methods are necessary if more details such as quantitative results of particle size and number density are required.

The same authors have performed, in the same environment, a microvacuum assessment adapting it from ASTM D5755 Standard test method for microvacuum sampling and indirect analysis of dust by transmission electron microscopy for asbestos structure number surface loading [30]. After having analysed the sampling media (a polycarbonate filter, included in a polypropylene cassette) by means of TEM/EDX analysis, the researchers found nanoparticles of Si and $\mathrm{Al}$ in five over nine samples.

The approach of tape sampling of surfaces has been proposed by Hedmer et al. who adopted a method conforming to the standardized one proposed by ASTM [31] in their study of a facility producing multi-walled carbon nanotubes [6], finding those nano structures, along with nano discs, in a significant amount of samples after SEM analysis (in 50\% and $17 \%$ of samples, respectively). Hedmer et al. concluded that tape sampling is a functional method for assessing surface contamination and exposure control during production at potentially any workplace that produces or handles carbon-based nano object and aggregates; it is also an important complementary method to air sampling, which can contribute to a better view of the hygienic situation of workplaces.

\section{Decontamination Techniques}

When the skin comes in contact with NPs the decontamination is not an easy issue. Due to the small size NPs can penetrate into the stratum corneum and the washing procedures can't be effective to remove the NPs that may remain on the surface of the skin and subsequently penetrate into the skin or reach the hair follicles [32]. For that reasons it is extremely important to avoid the skin contact with NPs using effective protective equipment.

\section{Biological Monitoring and Markers of NPs Exposure in Workers}

The air, skin and surfaces monitoring can be integrated by the research in biological fluids (urine, blood) or exhaled breath of markers of exposure or effects in exposed workers. When people are exposed to NPs containing metals, those metals can be search into urine or blood, using the biological monitoring suggested by the American Conference of Governmental Industrial Hygienist [33].

Liao et al. [34]. studied for 6 months workers of different factories exposed to nanomaterials in Taiwan. The group found that antioxidant enzymes (superoxide dismutase, glutathione peroxidase), cardiovascular markers (vascular cell adhesion molecule, paraoxonase), the small airway damage marker (Clara cell protein 16) and lung function test parameters were significantly associated with nanomaterial-handling.

In workers exposed to carbon nanotubes, Shvedova et al. [35] analysed changes in the ncRNA and mRNA expression profiles in blood, finding significant 
differences between subjects exposed for at least 6 months and controls.

Peclova et al. [36], in 2015, analyzed the content in titanium dioxide (anatase or rutile) in exhaled breath condensate of workers exposed to titanium dioxide nanoparticles finding a detectable amount of the metal in post shifts samples. No titanium was detectable in urine.

In workers exposed to iron oxide nanoparticles Peclova et al. [37], in 2016, found in exhaled breath condensate a significant increase of markers of oxidative stress such as malondialdehyde, 4-hydroxytrans-hexenale, 4-hydroxy-trans-nonenale, 8-isoProstaglandin F2 $\alpha$ (8-isoprostane) and aldehydes C6-C12 and markers of nucleic acid oxidation, such as 8-hydroxy-2-deoxyguanosine (8-OHdG), 8-hydroxyguanosine (8-OHG), 5-hydroxymethyl uracil (5-OHMeU).
The same authors, in another study [38], found a significant increase of markers of oxidative stress in exhaled breath condensate of workers exposed to titanium dioxide NPs.

\section{Assessment of NPs in Real Occupational Contexts: A Simplified Integrated Approach to Workplace Evaluation}

In the following table (Table 1) there is a summary of the instruments adopted in the assessment of nanoparticles in real occupational contexts.

Given all the above mentioned consideration, it is quite difficult to suggest a single and comprehensive approach to NPs evaluation in real workplace contexts, but we feel that a stepwise approach for risk assessment may be recommended (Fig. 3).

Table 1 Summary of the instruments adopted in the assessment of nanoparticles in real occupational contexts.

\begin{tabular}{|c|c|c|c|}
\hline Eval. & Tech. & Occupational contexts & Refs. \\
\hline Air & MPS & $\begin{array}{l}\text { Fullerene factory, processing of } \mathrm{TiO} 2, \mathrm{TiO} 2 \text { spray, silver, nanofibers, synthetic ceramic } \\
\text { nano-powders, carbon nanotube production and/or handling, refinery, highway } \\
\text { tollbooth, quantum dot spray deposition, fastener manufacturing plant, laser printers, } \\
\text { thermal cutting polystyrene foams, traditional pottery manufacturing, 3D Printer } \\
\text { Operation, iron oxide pigment production, welding fumes. }\end{array}$ & $\begin{array}{l}26,39,40,41,42,43,44,45,46 \\
47,48,49,50,51,52,53,38,37 \\
54,55\end{array}$ \\
\hline Air & OPC & $\begin{array}{l}\text { Laser printers, production of epoxy/carbon nanotubes and nanocomposites, fullerene } \\
\text { factory, traditional pottery manufacturing, carbon nanotube spraying process, recycling } \\
\text { facility }\end{array}$ & $40,43,50,56,57,58,59$ \\
\hline Air & СРC & $\begin{array}{l}\text { Offices (laser printer), semiconductor fabrication, production of epoxy/carbon } \\
\text { nanotubes and nanocomposites. }\end{array}$ & $26,27,2956,57,60$ \\
\hline Air & NSAM & $\begin{array}{c}\text { Fastener manufacturing process, graphene manufacturing, carbon black manufacturing } \\
\text { industry, welding operations }\end{array}$ & $25,47,61,62,63$ \\
\hline Air & APS & $\begin{array}{l}\text { Laser printers, production of carbon nanotubes, professional use of titanium dioxide, } \\
\text { iron oxide pigment production }\end{array}$ & 26, 38, 37, 41 \\
\hline Air & $\begin{array}{l}\text { Aerosol } \\
\text { monitor }\end{array}$ & Laser printers, carbon black manufacturing industry, carbon nanotube spraying process & $26,58,61$ \\
\hline Air & LPI & Rubber manufacturing factory, tire manufacturing facility & 28,29 \\
\hline Air & $\begin{array}{l}\text { Cascade } \\
\text { impactor }\end{array}$ & Carbon nanotube manufacturing, thermal cutting of polystyrene foams & 49, 55 \\
\hline Air & $\begin{array}{l}\text { Diffusion } \\
\text { charger }\end{array}$ & Laser printers, recycling facility & 26,59 \\
\hline Air & $\begin{array}{l}\text { Personal } \\
\text { samplers for } \\
\text { NPs }\end{array}$ & \multicolumn{2}{|c|}{$\begin{array}{l}\text { Welding fumes (Nanoparticle Respiratory Deposition sampler) [64]. Most of the available data on nanoparticles have been } \\
\text { tested in research or controlled /experimental environments, there is almost no data in real occupational contexts. }\end{array}$} \\
\hline Skin & Ad. TS & Multi-walled carbon nanotubes small-scale facility & 6 \\
\hline Surf. & Wipe sampling & Semiconductor production facility & 18 \\
\hline Surf. & $\begin{array}{l}\text { Micro- } \\
\text { vacuum }\end{array}$ & Semiconductor production facility & 18 \\
\hline Surf & Tape sampling & Multi-walled carbon nanotubes small-scale facility & 6 \\
\hline
\end{tabular}




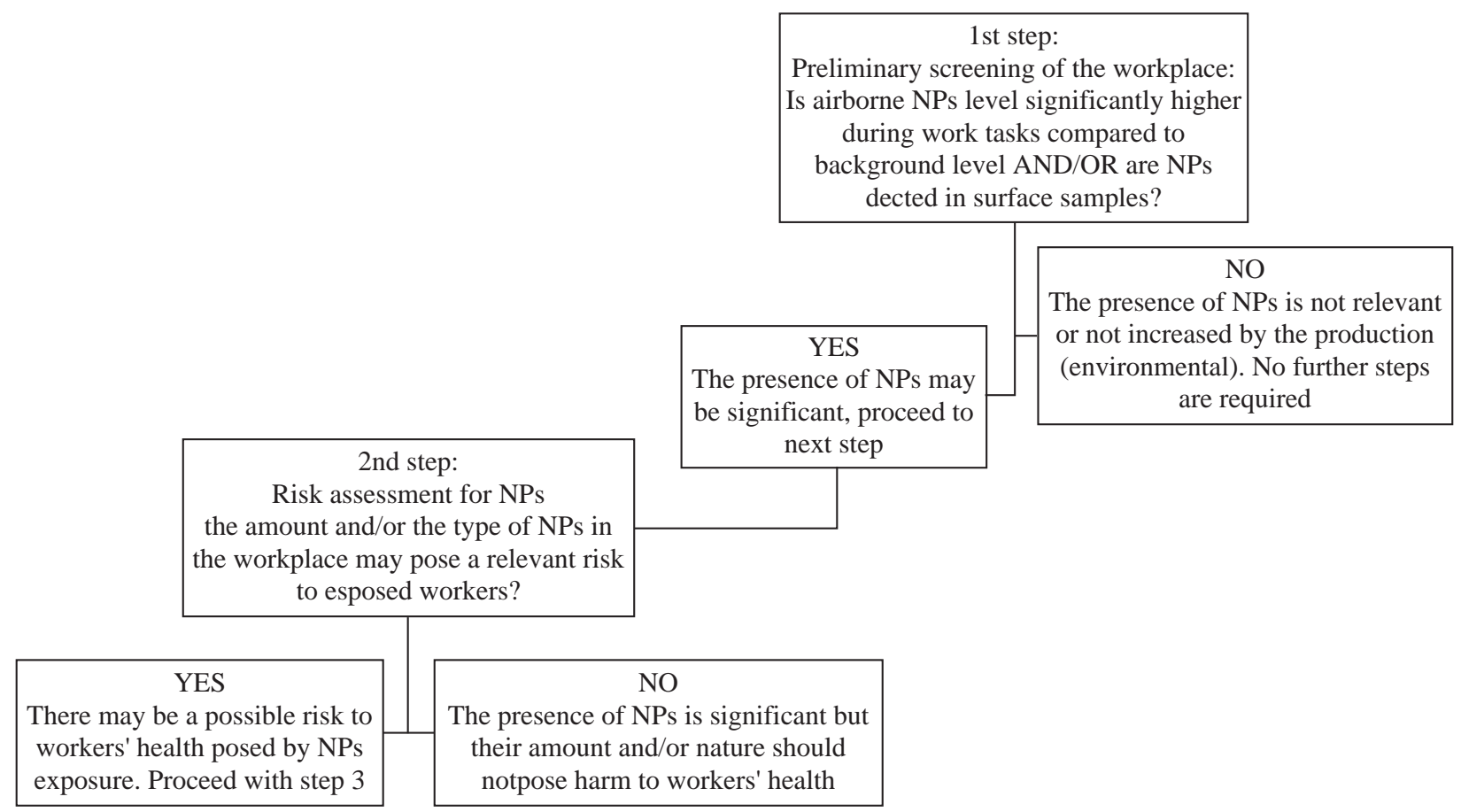

Fig. 3 A scheme / flowchart to the proposed stepwise approach to NPs occupational risk assessment.

1st step: preliminary screening of the workplace à the purpose of this step is to give a quick and low effort evaluation of the workplace in order to verify if there is the need to perform a risk assessment for NPs in the workplace (2nd step):

- Identify the possible sources of NPs, following the advices described in General recommendations prior to each assessment section.

- Using a portable nano-counter (e.g. DiSCMini) assess the baseline environmental presence of NPs in the workplace, when production is not running (e.g. before the beginning of the work shift).

- With the same tool, assess whether an increase of the presence of NPs occurs when production is going on, especially in close position to the main identified sources.

- Assess some of the workplace's surfaces, both close and far from the emission sources, using carbon tabs / tape strips and place some carbon pad in critical spots to catch airborne NPs; evaluate the presence and the composition of nanoparticles using electron microscopy and analytical methods such as EDX, in order to check if the NPs are actually related to the production cycle.

2nd step: risk assessment for NPs in the workplace à this step is important to qualify and quantify the risk for NPs in the examined occupational context:
- This step is recommended whether an increase in the presence of NPs is evidenced by portable nanocounters and/or if NPs are detected in carbon tabs / tape strips.

- Perform environmental and personal air sampling with the use of a proper impactor and sampling media in order to proceed with a TEM analysis coupled with EDX spectroscopy for a qualitative and morphological evaluation.

- Increase the number of carbon tabs and tape strips, sampling and analysing as described in 1st step. Wipe testing from the surfaces, along with the subsequent chemical analysis, can be performed in the same areas in order to increase the available information.

- Perform some tape strip from the skin of workers, and evaluate samples by means of electron microscopy.

3rd step: detailed assessment and characterization of NPs in the workplace à when required, this step is useful to further qualify and quantify the presence of NPs in the workplace:

- This step requires heavy, complex and expensive instrumentation and should be recommended only when a relevant risk is encountered at the 2nd step. Since at present the are no guidelines on risk evaluation for NPs, the evaluation of the relevance 
of the risk (especially considering the amount, composition and shape of the particles) should be made by personnel with a certain experience on health impact of NPs.

- One or more of the techniques described in table 1 for the air sampling of NPs (e.g. CPC, MPS, etc.) should be used in order to better identify the characteristics of airborne NPs.

- If needed, increase the number of available information from tape strips, carbon pads, wipe samples, etc.

- Perform biological monitoring to research metals in urine, blood or exhaled breath condensate. Evaluate effects in exhaled breath condensate.

Even though this stepwise approach does not guarantee a full characterization of NPs in the first two steps, it is a reasonable compromise in order to evaluate with cheaper techniques whether there actually is a relevant exposure to NPs and consequently the need to perform further evaluation with more sophisticated and expensive techniques.

\section{Conclusions}

The exposure of workers to NPs may follow several routes and there is neither an all-in-one solution nor a standardized method for a proper assessment or for the monitoring. It is required a good knowledge of the workplace and the production cycle before the assessment procedure.

There are several air monitoring strategies, some of which are re-adaptations of techniques already used for non-nano compounds while some other are specifically designed for NPs.

The skin may be assessed by means of interception methods (e.g. carbon pads), removal methods (such as skin wiping or adhesive tape stripping) or in situ methods. Similar techniques may be used in the assessment of the contamination of surfaces and equipment, along with microvacuum techniques.

Despite the advantages and the disadvantages of each method, further studies are needed, possibly in real occupational contexts, to further evaluate those techniques and/or to try new ones in order to develop a scientifically accepted protocol for risk assessment in the workplaces.

A stepwise approach to the evaluation of NPs in the workplace is proposed, in order to suggest a quick and relatively cheap screening method to assess exposure, followed by 2 gradually more accurate but more expensive approaches to perform whether there is a significant exposure to NPs.

\section{References}

[1] X. Dai, D. Cui, Advances in the toxicity of nanomaterials. Nano Biomed Eng, 2012, 4(3): 150-156.

[2] X. Yu, F. Hong and Y. Zhang, Bio-effect of nanoparticles in the cardiovascular system. J Biomed Mater Res, 2016, doi: 10.1002/jbm.a.35804.

[3] L. Ding, Z. Liu, M. Aggrey, et al., Nanotoxicity: the toxicity research progress of metal and metal-containing nanoparticles. Mini Rev Med Chem, 2015, 15(7): 529-542.

[4] S. Bakand, A. Hayes, Toxicological considerations, toxicity assessment and risk management of inhaled nanoparticles. Int J Mol Sci, 2016, 17(6): 926.

[5] F. Larese Filon, M. Mauro, G. Adami, et al., Nanoparticles skin absorption: new aspects for a safety profile evaluation. Regul Toxicol Pharmacol, 2015, 72(2): 310322.

[6] M. Hedmer, L. Ludvigsson, C. Isaxon, et al., Detection of multi-walled carbon nanotubes and carbon nanodiscs on workplace surfaces at a small-scale producer. Ann Occup Hyg, 2015, 59(7): 836-852.

[7] National Institute of Health - Office of Research Services - Division of Occupational Health and Safety, Nanotechnology safety and health program. 2014, Retrieved from http://www.ors.od.nih.gov/sr/dohs/ Documents/Nanotechnology\%20Safety\%20and\%20 Health\%20Program.pdf.

[8] M. Debia, C. Beaudry, S. Weichenthal, et al., Characterization and control of occupational exposure to nanoparticles and ultrafine particles. Chemical substances and biological agents, studies and reserch projects, IRSST - Institut de recherche Robert-Sauvé en santé et en sécurité du travail, 2013.

[9] C. Ostiguy, B. Roberge, L. Ménard, et al., Guide de bonnes pratiques favorisant la gestion des risques reliés aux nanoparticules de synthèse. Substances chimiques et agents biologiques, études et recherches, Institut de recherche Robert-Sauvé en santé et en sécurité du travail, 2008: 63.

[10] D. Nash, D. Leith, Ultrafine particle sampling with the UNC passive aerosol sampler. Aerosol Science and Technology, 2010, 44(12): 1059-1064.

[11] J. Marra, M. Voetz and H. Kiesling, Monitor for detecting and assessing exposure to airborne nanoparticles. Journal of Nanoparticle Research, 2010, 12(1): 21-37.

[12] A. Khan, A. Mishra, P. Pandey, et al., Measurement techniques and instruments for airborne nanoparticles. Journal of Biomedical Nanotechnology, 2011, 7(1): 165.

[13] M. Fierz, C. Houle, P. Steigmeier, et al., Design, calibration, and field performance of a miniature diffusion size classifier. Aerosol Science and Technology, 2011, 45(1): 1-10.

[14] M. Fierz, A. Keller and H. Burtscher, Charge-based personal aerosol samplers. Inhalation Toxicology, 2009, 21(S1): 30-34.

[15] A. Fonseca, M. Viana and X. Querol, Ultrafine and nanoparticle formation and emission mechanisms during laser processing of ceramic materials. Journal of Aerosol Science, 2015, 88: 48-57.

[16] G. Bartolucci, M. Carrieri and D. Cottica. Strumenti e tecniche dell'Igiene Industriale: il monitoraggio 
ambientale. Trattato di Medicina del Lavoro, Piccin, 2015: 153-157.

[17] S. Semple, Dermal exposure to chemicals in the workplace: just how important is skin absorption? Occup Environ Med, 2004, 48: 376-382.

[18] M. Shepard, S. Brenner, Cutaneous exposure scenarios for engineered nanoparticles used in semiconductor fabrication: a preliminary investigation of workplace surface contamination. Int J Occup Environ Health, 2014, 20(3): 247-257.

[19] W. Popendorf, J. Leffingwell, Regulating OP pesticide residues for farmworker protection. Res Rev, 1982, 82: 125-201.

[20] E. Gehan, S. George, Estimation of human body surface area from height and weight. Cancer Chemother Rep, 1970, 54: 225-235.

[21] D. Brouwer, M. Boeniger and J.V. Hemmen, Hand wash and manual skin wipes. Ann Occup Hyg, 2000, 44(7): 501-510.

[22] ASTM International, ASTM D6966-08 Standard practice for collection of settled dust samples using wipe sampling methods for subsequent determination of metals. Annual Book of ASTM Standards, 2008.

[23] J. Lademann, U. Jacobi, C. Surber, et al., The tape stripping procedure - evaluation of some critical parameters. Eur J Pharm Biopharm, 2009, 72(2): 317323.

[24] CEN/TC 137/WG 6, Draft CEN/TS Workplace exposure Assessment of dermal exposure to nano-objects and their aggregates and agglomerates (NOAA). ISO Standards catalogue, 2015.

[25] J. Lee, J. Han, J. Kim, et al., Exposure monitoring of graphene nanoplatelets manufacturing workplaces. Inhal Toxicol, 2016, 8: 1-11.

[26] J. Niu, P. Rasmussen, R. Magee, et al., Spatial and temporal variability of incidental nanoparticles in indoor workplaces: impact on the characterization of point source exposures. Environ Sci Process Impacts, 2015, 17(1): 98109.

[27] M. Shepard, S. Brenner, An occupational exposure assessment for engineered nanoparticles used in semiconductor fabrication. Ann Occup Hyg, 2014, 58(2): 251-265.

[28] M. Kreider, W. Cyrs, M. Tosiano, et al., Evaluation of quantitative exposure assessment method for nanomaterials in mixed dust environments: application in tire manufacturing facilities. Ann Occ Hyg, 2015, 59(9): $1122-1134$.

[29] B. Kim, J. Lee, B. Choi, et al., Ultrafine particle characteristics in a rubber manufacturing factory. Ann Occup Hyg, 2013, 57(6): 728-739.

[30] ASTM International, ASTM D6480-05(2010) Standard test method for wipe sampling of surfaces, indirect preparation, and analysis for asbestos structure number concentration by transmission electron microscopy. Annual Book of ASTM Standards, 2010: 11.07.

[31] ASTM International, ASTM E1216-11 Standard practice for sampling for particulate contamination by tape lift. Annual Book of ASTM Standards, 2011: 15.03.

[32] M. Ovissipour, S. Sablani and B. Rasco, Engineered nanoparticle adhesion and removal from tomato surfaces. J Agric Food Chem, 2013, 61(42): 10183-10190.

[33] ACGIH®, TLV® ${ }^{\circledR}$ BEI ${ }^{\circledR}$ guidelines. Retrieved from http:// www.acgih.org/tlv-bei-guidelines/policies-procedurespresentations/overview.

[34] H. Liao, Y. Chung, C. Lai, et al., Six-month follow-up study of health markers of nanomaterials among workers handling engineered nanomaterials. Nanotoxicology, 2014, Suppl.1: 100-110.

[35] A. Shvedova, N. Yanamala, E. Kisin, et al., Integrated analysis of dysregulated ncRNA and mRNA expression profiles in humans exposed to carbon nanotubes. PLoS ONE, 11(3): e0150628.

[36] D. Pelclova, H. Barosova, J. Kukutschova, et al., Raman microspectroscopy of exhaled breath condensate and urine in workers exposed to fine and nano $\mathrm{TiO} 2$ particles: a cross-sectional study. J Breath Res, 2015, 9(3): 036008.

[37] D. Pelclova, V. Zdimal, P. Kacer, et al., Oxidative stress markers are elevated in exhaled breath condensate of workers exposed to nanoparticles during iron oxide pigment production. J Breath Res, 2016, 10(1):016004.

[38] D. Pelclova, V. Zdimal, Z. Fenclova, et al., Markers of oxidative damage of nucleic acids and proteins among workers exposed to $\mathrm{TiO} 2$ (nano) particles. Occup Environ Med, 2016, 73(2): 110-118.

[39] S. Plitzko, Workplace exposure to engineered nanoparticles. Inhal Toxicol, 2009, 1(Suppl 1): 25-29.

[40] Y. Fujitani, T. Kobayashi, K. Arashidani, et al., Measurement of the physical properties of aerosols in a fullerene factory for inhalation exposure assessment. $J$ Occup Environ Hyg, 2008, 5(6): 380-389.

[41] S. Tsai, M. Hofmann, M. Hallock, et al., Characterization and evaluation of nanoparticle release during the synthesis of single-walled and multiwalled carbon nanotubes by chemical vapor deposition. Environ Sci Technol, 2009, 43(15): 6017-6023.

[42] A. Miller, P. Drake, P. Hintz, et al., Characterizing exposures to airborne metals and nanoparticle emissions in a refinery. Ann Occup Hyg, 2010, 54(5): 504-513.

[43] M. Takaya, F. Serita, M. Ono-Ogasawara, et al., Airborne particles in a multi-wall carbon nanotube production plant: observation of particle emission and personal exposure 1: measurement in the packing process. Sangyo Eiseigaku Zasshi, 2010, 52(4): 182-188.

[44] J. Lee, M. Kwon, J. Ji, et al., Exposure assessment of workplaces manufacturing nanosized $\mathrm{TiO}_{2}$ and silver. Inhal Toxicol, 2011, 23(4): 226-236.

[45] Y. Cheng, C. Huang, H. Huang, Concentrations of ultrafine particles at a highway toll collection booth and exposure implications for toll collectors. Sci Total Environ, 2010, 409(2): 364-369.

[46] J. Jankovic, S. Hollenbeck and T. Zontek, Ambient air sampling during quantum-dot spray deposition. Int $J$ Occup Environ Health, 2010, 16(4): 388-398.

[47] Y. Wang, P. Tsai, C. Chen, et al., Size distributions and exposure concentrations of nanoparticles associated with the emissions of oil mists from fastener manufacturing processes. J Hazard Mater, 2011, 198: 182-187.

[48] P. Castellano, S. Canepari, R. Ferrante, et al., Multiparametric approach for an exemplary study of laser printer emissions. J Environ Monit, 2012, 14(2): 446-454.

[49] H. Zhang, Y. Kuo, A. Gerecke, et al., Co-release of hexabromocyclododecane (HBCD) and nano- and microparticles from thermal cutting of polystyrene foams. Environ Sci Technol, 2012, 46(20): 10990-10996.

[50] A. Voliotis, S. Bezantakos, M. Giamarelou, et al., Nanoparticle emissions from traditional pottery manufacturing. Environ Sci Process Impacts, 2014, 16(6): 1489-1494.

[51] W. Heitbrink, L. Lo and K. Dunn, Exposure controls for nanomaterials at three manufacturing sites. J Occup Environ Hyg, 2015, 12(1): 16-28.

[52] J. Lee, K. Ahn and S. Kim, Three-day continuous exposure monitoring of CNT manufacturing workplaces. Biomed Res Int, 2015, 2015: 237140.

[53] Y. Kim, C. Yoon, S. Ham, et al., Emissions of nanoparticles and gaseous material from 3D printer operation. Environ Sci Technol, 2015, 49(20): $12044-$ 12053.

[54] B. Chen, D. Schwegler-Berry, A. Cumpston, Performance 
of a scanning mobility particle sizer in measuring diverse types of airborne nanoparticles. Multi-walled carbon nanotubes, welding fumes, and titanium dioxide spray. $J$ Occup Environ Hyg, 2016, 13(7): 501-518.

[55] J. Lee, S. Lee, G. Bae, et al., Exposure assessment of carbon nanotube manufacturing workplaces. Inhal Toxicol, 2010, 22(5): 369-381.

[56] M. Grana, L. Vicentini, A. Pietroiusti, et al., Ultrafine particle emissions from laser printers. G Ital Med Lav Ergon, 2015, 37(3): 135-143.

[57] L. Cena, T. Peters, Characterization and control of airborne particles emitted during production of epoxy/ carbon nanotube nanocomposites. J Occup Environ Hyg, 2011, 8(2): 86-92.

[58] J. Ji, D. Woo, S. Lee, et al., Detection and characterization of nanomaterials released in low concentrations during multi-walled carbon nanotube spraying process in a cleanroom. Inhal Toxicol, 2013, 25(14): 759-765.

[59] P. Raynor, J. Cebula, J. Spangenberger, et al., Assessing potential nanoparticle release during nanocomposite shredding using direct-reading instruments. J Occup Environ Hyg, 2012, 9(1): 1-13.

[60] K. Choi, J. Kim, J. Park, et al., Exposure characteristics of nanoparticles as process by-products for the semiconductor manufacturing industry. J Occup Environ
Hyg, 2015, 12(8): 153-160.

[61] Y. Wang, P. Tsai, C. Chen, et al., Using a modified electrical aerosol detector to predict nanoparticle exposures to different regions of the respiratory tract for workers in a carbon black manufacturing industry. Environ Sci Technol, 2010, 44(17): 6767-6774.

[62] J. Gomes, P. Albuquerque, R. Miranda, et al., Determination of airborne nanoparticles from welding operations. J Toxicol Environ Health A, 2012, 75(13-15): 747-755.

[63] J. Gomes, P. Albuquerque, R. Miranda, et al., Comparison of deposited surface area of airborne ultrafine particles generated from two welding processes. Inhal Toxicol, 2012, 24(11): 774-781.

[64] L. Cena, M. Keane, W. Chisholm, A novel method for assessing respiratory deposition of welding fume nanoparticles. J Occup Environ Hyg, 2014, 11(12): 771780 .

Copyright $₫ 2016$ Andrea Prodi, Francesca Larese Filon. This is an open-access article distributed under the terms of the Creative Commons Attribution License, which permits unrestricted use, distribution, and reproduction in any medium, provided the original author and source are credited. 